\section{Why carrot and stick doesn't work: motivation is an inside job}

\author{
Alan Robinson
}

'Can you come and motivate my team?'

'Sure. Motivate them to do what? Trouble is that they are already highly motivated, dynamic, inspired and focused people - just not for the 8 hours they're at work!'

Motivating the team seems to be the most common and most difficult job of a practice manager. If it was easy we' $d$ all be doing it but there are so many demotivated and uninspired people in practices it begs the question is motivation possible - can you motivate other people or is it actually an inside-out job? If so, what can the manager do to create an environment that at least allows internal motivation to shine through? The new science of motivation sheds some light on this strange phenomenon.

Scientists have long known that two main drives power human behaviour - the biological drive, including hunger, thirst and sex, and the more scientific rewardpunishment motivation theory.

When work consisted largely of simple, uninteresting (industrial) tasks, in order to get as much productivity out of your workers as possible, you had to reward the behaviour you sought, and punish the behaviour you discouraged - the carrot-and-stick approach.

But jobs in the 21st century have changed dramatically. They have become more complex, more interesting and more self-directed, and this is where the carrot-and-stick approach has become unstuck. This traditional approach can result in:

\section{- Diminished intrinsic motivation \\ - Lower performance \\ - Less creativity \\ - 'Crowding out' of good behaviour \\ - Unethical behaviour \\ - Addictions and short-term thinking.}

This led to the discovery of a possible third drive for intrinsic motivation, the joy of the task itself - that human beings have an 'inherent tendency to seek out novelty and challenges, to extend and exercise their capabilities, to explore, and to learn'. This new theory of motivation proposes that businesses should adopt a revised approach to motivation which fits more closely with modern jobs and businesses, one based on self-determination theory (SDT). SDT proposes that human beings have an innate drive to be autonomous, self-determined and connected to one another, and that when that drive is liberated, people achieve more and live richer lives.

Practices should focus on these drives when managing their teams by creating settings which focus on our innate need to direct our own lives (autonomy), to learn and create new things (mastery), and to do better by ourselves and our world (purpose).
- Autonomy - provide employees with autonomy over some (or all) of the four main aspects of work:

- When they do it (time)

- How they do it (technique)

- Whom they do it with (team)

- What they do (task)

- Mastery - allow employees to become better at something that matters to them:

- Provide 'Goldilocks tasks' - those tasks which are neither overly difficult nor overly simple

- Create an environment where mastery is possible

- Communicate the purpose

- Place equal emphasis on purpose maximization as you do on profit maximization - Use purpose-oriented words such as 'us' and 'we'

- Purpose - take steps to fulfil employees' natural desire to contribute to a cause greater and more enduring than themselves.

The notion of increasing employee satisfaction through the intrinsic motivational methods of autonomy, mastery and purpose has obvious implications for remuneration models and incentive schemes traditionally used by practices.

\section{KEY LEARNING OBJECTIVES}

- Why traditional motivation theory doesn't work

- Introducing a new theory of intrinsic motivation

- Three drives of intrinsic motivation and their application in practice

\section{MULTIPLE CHOICE QUESTIONS}

1. Which of the following does the traditional 'carrot-and-stick' approach to motivation for complex tasks NOT result in?

(A) Increased intrinsic motivation

(B) Lower performance and less creativity

(C) 'Crowding out' of good behaviour and unethical behaviour

(D) Addictive behaviour and short-term thinking

2. What are team engagement and motivation driven by?

(A) Strict rules and protocols dictating behaviour

(B) An intrinsic sense of legacy, autonomy and mastery

(C) Extrinsic rewards such as bonuses, money and time off

(D) Removing all stress and pressure to perform well

3. Which of the following does NOT describe people who are experiencing processes that can increase intrinsic as distinct from extrinsic motivation?
(A) Are autonomous and self-determined
(B) Are connected to one another and cooperate
(C) Achieve more and live richer lives
(D) Require more money and incentives to work 